\title{
Half-filled orbital and unconventional geometry of a common dopant in $\operatorname{Si}(001)$
}

\author{
K. Iwaya, ${ }^{1, *}$ D. R. Bowler, ${ }^{1,2}$ V. Brázdová, ${ }^{1,2}$ A. Ferreira da Silva, ${ }^{3}$ Ch. Renner,${ }^{4}$ W. Wu,${ }^{1,2}$ A. J. Fisher ${ }^{1,2}$ \\ A. M. Stoneham, ${ }^{1,2}$ and G. Aeppli ${ }^{1}$ \\ ${ }^{1}$ London Centre for Nanotechnology and Department of Physics and Astronomy, University College London, \\ Gordon Street, London WCIH OAH, United Kingdom \\ ${ }^{2}$ TYC@UCL, University College London, Gower Street, London WC1E 6BT, United Kingdom \\ ${ }^{3}$ Institute of Physics, Federal University of Bahia, 40210340 Salvador, Bahia, Brazil \\ ${ }^{4}$ Department of Condensed Matter Physics, University of Geneva, Geneva, CH-1211, Switzerland
}

(Received 11 February 2013; published 26 July 2013)

\begin{abstract}
The determining factor of the bulk properties of doped $\mathrm{Si}$ is the column rather than the row in the periodic table from which the dopants are drawn. It is unknown whether the basic properties of dopants at surfaces and interfaces, steadily growing in importance as microelectronic devices shrink, are also solely governed by their column of origin. The common light impurity P replaces individual Si atoms and maintains the integrity of the dimer superstructure of the $\mathrm{Si}(001)$ surface, but loses its valence electrons to surface states. Here we report that isolated heavy dopants are entirely different: $\mathrm{Bi}$ atoms form pairs with $\mathrm{Si}$ vacancies, retain their electrons, and have highly localized, half-filled orbitals.
\end{abstract}

DOI: 10.1103/PhysRevB.88.035440

PACS number(s): 61.72.uf, 68.35.bg, 71.55.-i

\section{INTRODUCTION}

Semiconductors such as silicon $(\mathrm{Si})$ derive remarkable utility from the ease with which they are converted from insulating to conducting behavior via substitution of dopants from neighboring columns in the periodic table. Isolated phosphorus (P) and bismuth (Bi) atoms, both with one more valence electron than $\mathrm{Si}$, substitute for $\mathrm{Si}$ atoms, and behave approximately as hydrogenic atoms with radii and binding energies described by the Bohr theory where the electron mass and dielectric constant are those provided by the diamond lattice of Si rather than the vacuum.

Dopants in the bulk are well understood, but their properties at surfaces are poorly understood; for instance, we have recently shown that heavy dopants at $\mathrm{Si}(111)$ can induce ambipolar charge states. ${ }^{1}$ There is growing interest in heavier dopants of conventional semiconductors because of their more tightly bound outer electrons, which increases operating temperatures for applications ranging from $\mathrm{THz}$ impurity lasers to quantum computers exploiting their spin. ${ }^{2}$ We have accordingly chosen the heaviest column $\mathrm{V}$ element for the current study. Here we report that isolated heavy dopants in the $\mathrm{Si}(001)$ surface are entirely different to light dopants: $\mathrm{Bi}$ atoms form pairs with $\mathrm{Si}$ vacancies, retain their electrons and have highly localized, half-filled orbitals. Thus, we finally have a dopant of silicon with a half-filled orbital at the surface, enabling both new applications in spintronics, as well as $\mathrm{Si}$ surface-based quantum many-body physics, hitherto confined to the more complex $\mathrm{Si}(111)$ surface.

\section{METHODS}

Our samples are semiconductor grade $p$-type $\mathrm{Si}(001)$ wafers (with boron [B] doping of $\sim 10^{15} \mathrm{~cm}^{-3}$ ) implanted with $\mathrm{Bi}^{+}$ions $(\mathrm{Si}: \mathrm{Bi})$. The $\mathrm{Bi}$ donor concentration estimated from transport measurements is $\sim 2.2 \times 10^{19} \mathrm{~cm}^{-3}$, which is near the metal-nonmetal transition. ${ }^{3}$

Scanning tunneling microscopes (STMs), where a metallic tip is positioned to sub-Angstrom precision using piezoelectric drives and the tunneling current between tip and sample is directly measured and/or used to control the drives, are uniquely suited to determine the electronic structure of surfaces. Here we employ an Omicron low-temperature (LT)STM, at $5 \mathrm{~K}$ in ultrahigh vacuum (UHV) with a base pressure of $\sim 4 \times 10^{-9} \mathrm{~Pa}$. For the $\mathrm{Si}(001)$ surface, strong tip-surface interactions can induce a complicated, unstable structural change between $c(4 \times 2)$ and $p(2 \times 2)$ reconstruction at low temperature. ${ }^{4,5}$ To avoid this, we ensure a relatively large tip-sample separation during measurements by using small tunneling currents, typically $100 \mathrm{pA}$ for topographical imaging.

The surface was prepared in UHV by a standard flashing (direct current heating) method at $\sim 1100^{\circ} \mathrm{C}$ for 10 seconds. The pressure was kept below $1 \times 10^{-8}$ Pa during the flashing to prepare a clean surface. When searching for features resulting from $\mathrm{Bi}$ dopants, we must first eliminate other features. The electronic structure of standard defects in a clean $\mathrm{Si}(001)$ surface has already been studied by STM $^{6-8}$ and so such defects can be identified and eliminated from our study by bias-voltage-dependent imaging. The native $\mathrm{B}$ dopant might also be observed; however, it has a much lower density than the ion-implanted Bi impurities, and bias-dependent STM images of a single $\mathrm{B}$ dopant in $\mathrm{Si}(001)$ surface have already been reported, ${ }^{9}$ so also allowing $\mathrm{B}$ impurities to be identified, if present, and excluded from the current study.

The electronic structure calculations used density functional theory (DFT) within the PBE generalized gradient approximation (GGA) ${ }^{10}$ The system was modeled with a slab at least six layers deep with the last two layers fixed in bulklike positions and the bottom layer terminated in hydrogen. The Brillouin zone was sampled with the same density for all cells at a level which gave meV convergence in total energies. We used two different basis sets and codes: plane waves and Gaussians.

The plane-wave basis calculations as implemented in the VASP $\operatorname{code}^{11}$ used a cutoff energy of $200 \mathrm{eV}$, which converged energy differences to meV accuracy. We used ultrasoft pseudopotentials ${ }^{12}$ and spin-polarized density 
functional theory, with the PBE GGA functional ${ }^{10}$ for the exchange-correlation terms. After performing spin-polarized calculations we also tested the effect of including spin-orbit interactions, which made only a small difference to the electronic structure. For calculations on a single dopant, we chose the cell sizes to vary the spacing between its periodic images. We used cells with between 1 and 3 dimer rows, giving lateral sizes of $0.77,1.54$, and $2.30 \mathrm{~nm}$, with four to eight dimers in the cell, giving lengths of 1.54 and 3.07 $\mathrm{nm}$. The basic density of $k$ points was set by the $1.54 \mathrm{~nm} \times$ $1.54 \mathrm{~nm}$ cell, which we sampled with a mesh of $4 \times 4 \times 1$ $k$ points; other cells had the number of $k$ points varied to achieve the same density, and convergence checks were made on all cells. Structural relaxations were carried out until all components of all forces were less than $0.25 \mathrm{eV} / \mathrm{nm}$.

Exact exchange is relatively inefficient to implement in plane-wave DFT because the exchange kernel is nonlocal; we therefore use the CRSYTAL06 code, ${ }^{13}$ which has a Gaussian basis set, using the same atomic coordinates as were found by the VASP calculations. The basis sets used were $88-31 \mathrm{G}^{\star}$ for $\mathrm{Si}$ and $\mathrm{Bi}$ and $31 \mathrm{G}$ for the terminating $\mathrm{H}$ atoms on the reverse of the slab, with an all-electron treatment of the Si atoms and an effective core potential ${ }^{14}$ for the $\mathrm{Bi}$ atoms. The $k$-point sampling was a $(2 \times 1 \times 1)$ mesh, giving the same density as in the VASP calculation.

\section{RESULTS}

\section{A. Atomic structure}

The $\operatorname{Si}(001)$ surface shows characteristic $\langle 110\rangle$ rows of dimerized $\mathrm{Si}$ atoms. ${ }^{15}$ Dimers along the row are separated by $a_{0} / \sqrt{2}=0.38 \mathrm{~nm}$, where $a_{0}$ is the cubic lattice constant of Si. Tunneling images of the Bi-implanted samples after in-situ surface preparation contained four easily distinguished features attributable to Bi donors. The bismuth-vacancy feature we describe in this work, referred to as $\mathrm{Bi}-\mathrm{V}$, is located at the surface; we have found other features which are clearly subsurface features and will be discussed in a future publication; these subsurface donors are considerably less common than the surface Bi- $\mathrm{V}$ pairs. Of our large inventory of 500 observed features, $\sim 84 \%$ are Bi-V pairs and are located at the surface. Figures 1(a) and 1(b) display emptyand filled-state topographs of a single Bi-V defect and how it fits within the dimer rows. For filled states (Fig. 1(b); a sample-bias-voltage $\mathrm{V}_{S}$ of $-2 \mathrm{~V}$ ) the $\mathrm{Bi}-\mathrm{V}$ feature is similar to the surrounding surface, though a little brighter. For empty states [Fig. 1(a); $\mathrm{V}_{S}=+2 \mathrm{~V}$ ], it appears as a bright protrusion neighboring a depression surrounded by the characteristic dimer $\pi^{\star}$-bonding states; this is a very unusual structure in the $\mathrm{Si}(001)$ surface. The apparent flatness of the surface surrounding the $\mathrm{Bi}-\mathrm{V}$ feature indicates that the $\mathrm{Bi}$ is not charged - strong brightening is seen around charged dopants in $\operatorname{Si}(001),{ }^{16}$ due to band bending. Considering the topographic structure, it is extremely likely that the Bi atom substitutes for a $\mathrm{Si}$ atom in the surface, as illustrated in Fig. 1(c).

As a further check on the identity of the new features we investigated the stability of the Bi-V structure and its population density by repeated sample flashing. The density
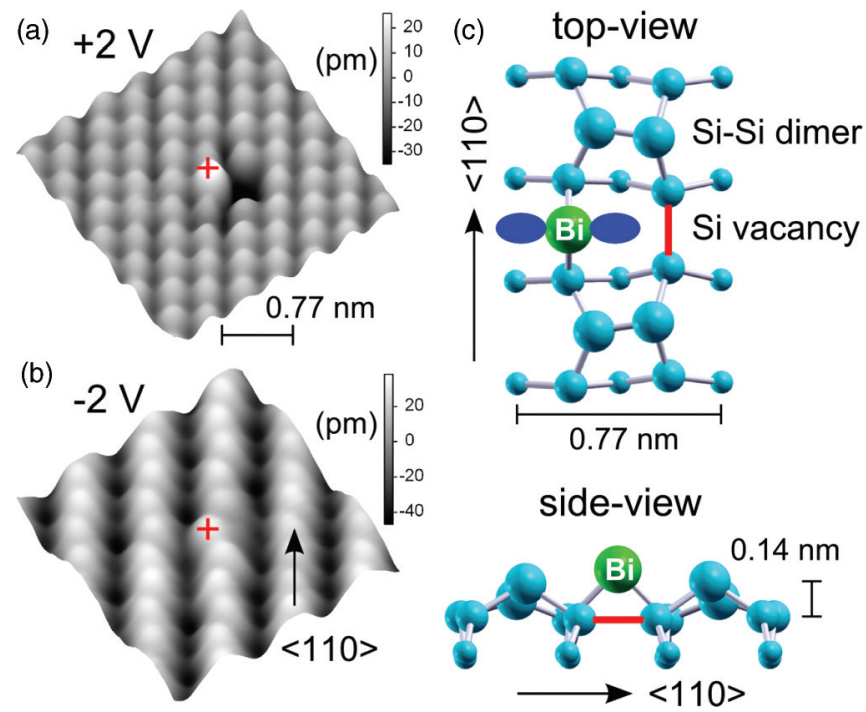

FIG. 1. (Color online) Structure of Bi-Si vacancy complex in the $\mathrm{Si}(001)$ surface. (a),(b) Bias-dependent topographic images of $\mathrm{Bi}$ donor in the $\mathrm{Si}(001)$ surface at sample-bias voltages of $\mathrm{V}_{s}=$ $+2 \mathrm{~V}$ (empty state) and $-2 \mathrm{~V}$ (filled state), respectively. The tunneling current was $\mathrm{I}_{t} \sim 100 \mathrm{pA}$. Bi sites are indicated by markers. The color scale is in units of pm. The horizontal scale is indicated in (a) by the spacing between $\mathrm{Si}-\mathrm{Si}$ dimer rows $(\sim 0.77 \mathrm{~nm})$. (c) Schematic structure of Bi-Si vacancy complex in the $\mathrm{Si}(001)$ surface (top-, side-view). The neutral $\mathrm{Bi}$ atom has a half-filled $p$ orbital perpendicular to the Si dimer row, and at the Si vacancy site the second-layer $\mathrm{Si}$ atoms bond across the vacancy as shown by a dotted line.

is estimated from topographic images [Fig. 2(a)] to be $\sim 2 \times 10^{11} \mathrm{~cm}^{-2}$ after repetitive surface flashing [Fig. 2(b)]. If we assume that $\mathrm{Bi}$ atoms in the first two layers are visible in STM, and have the same density as produced by the initial ion implantation/annealing protocols, then we expect to see $\left(2.2 \times 10^{19} \mathrm{~cm}^{-3}\right) \times(0.14 \mathrm{~nm}) \sim 3 \times 10^{11} \mathrm{~cm}^{-2}$ [as Si layer spacing along (001) is $0.14 \mathrm{~nm}$ ]. This is in excellent agreement with the defect density observed by STM, strengthening our identification of these features as $\mathrm{Bi}$ donors. Moreover, the
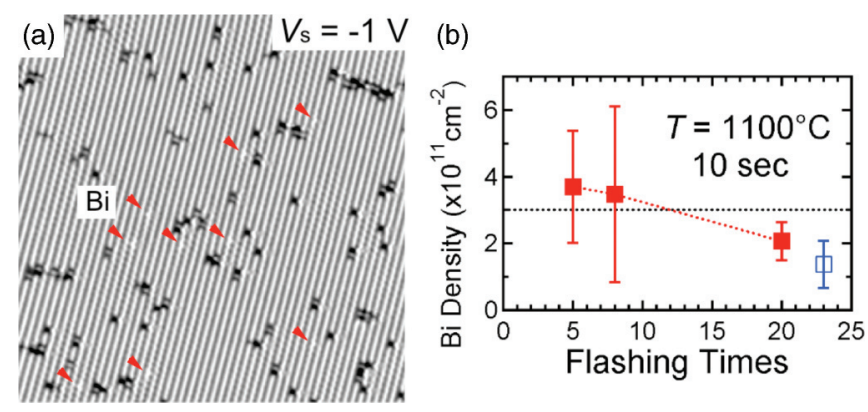

FIG. 2. (Color online) (a) Typical topographic image of $\mathrm{Si}(001)$ : Bi at $\mathrm{V}_{S}=-1 \mathrm{~V}$. Scan size is $40 \mathrm{~nm} \times 40 \mathrm{~nm}$. Bi donors are indicated by arrows. (b) Flashing effect on the density of Bi dopants in STM image. The flashing was performed at $1100^{\circ} \mathrm{C}$ for $10 \mathrm{sec}$. The sample was exposed to air after 20th flashing intentionally, and flashed again in UHV condition (blue square). For comparison, the horizontal dashed line represents the Bi concentration estimated from the bulk density $\left(\sim 3 \times 10^{11} \mathrm{~cm}^{-2}\right)$. 
Bi-V structure is always found, with a population density within a factor of two of that associated with the bulk dopant density. Even after the sample was exposed to air intentionally and heated again, the same defects are seen, although with a barely significant trend towards lower density with repeated surface treatments.

To identify the structure of the feature and further characterize it, we have performed total energy spin-polarized density functional theory (DFT) calculations. As the structure clearly involves a vacancy and the prepared surface contains a certain number of vacancies, we have considered structures involving a vacancy; the energetics of structures in the absence of vacancies have also been considered and are given below. Various candidate structures were considered: a missing dimer vacancy (1DV) and a mixed Bi-Si dimer with different charge states, by analogy with the P-Si heterodimer system; ${ }^{16-18}$ a $\mathrm{Bi}$ adatom on the $\mathrm{Si}(001)$ surface with a $1 \mathrm{DV}$; a $\mathrm{Bi}$ atom symmetrically replacing an entire $\mathrm{Si}$ dimer in the surface; and a Bi-vacancy pair replacing a $\mathrm{Si}$ dimer in the surface, again with different charge states. Kinetic effects during sample preparation produce an abundance of nonequilibrium structures. ${ }^{19}$ Accordingly, the main criterion used to judge the fitness of the structure was the agreement of the appearance between simulated and real STM images, rather than total energy from DFT; the stability of different features is discussed further below.

The only structure which accounts for the STM images is the neutral surface Bi-vacancy pair (Bi-V). Figure 1(c) is the associated schematic, while Fig. 3(a) allows comparison of STM topographic images and DFT calculations. In this model, one atom of a surface Si dimer is replaced by a twofold coordinated $\mathrm{Bi}$ atom, while the other $\mathrm{Si}$ atom is removed, and the second-layer $\mathrm{Si}$ atoms rebond across the vacancy as indicated by a red line in Fig. 1(c). The resulting Bi atom has a neutral configuration with two $6 s$ and three $6 p$ electrons, (a)

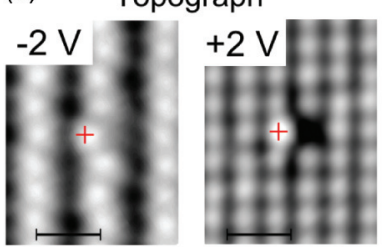

Integrated DOS map
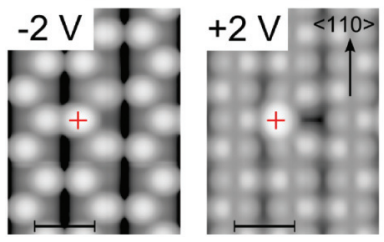

(b) $\quad d l / d V$ map

DOS map

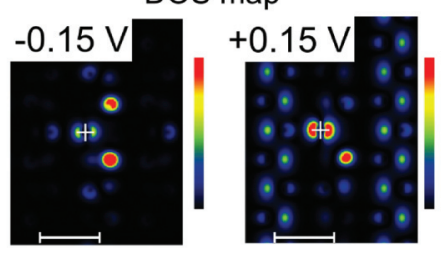

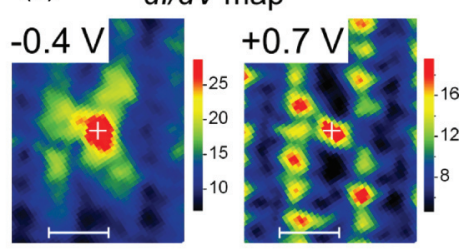

FIG. 3. (Color online) Comparison between STM/STS and spinpolarized DFT. (a) STM images (top) and simulated STM (bottom) of the Bi donor (plus sign). STM images were taken at exactly the same location; simulated images used isosurface values of 0.1 electron $/ \mathrm{nm}^{3}$. (b) $\mathrm{dI} / \mathrm{dV}$ maps (top) and simulated DOS maps around $\mathrm{Bi}-\mathrm{Si}$ vacancy complex (bottom). Feedback set-point was $\sim 100 \mathrm{pA}$, $\mathrm{V}_{S}=-2 \mathrm{~V}$. The spacing between $\mathrm{Si}-\mathrm{Si}$ dimer rows $(\sim 0.77 \mathrm{~nm})$ is shown in all images. The units of color scale in the $\mathrm{dI} / \mathrm{dV}$ map and DOS map are $\mathrm{pS}$ and electron $/ \AA^{3}$. unhybridized, in contrast to that in bulk $\mathrm{Si}$ where the donor ionizes leaving four electrons in $s p^{3}$ hybrid orbitals. This structure, and other unusual Bi structures at the $\mathrm{Si}(001)$ surface such as Bi nanolines, ${ }^{20}$ can be explained by considering the large energy difference between the $6 s$ and $6 p$ Bi valence electrons: ${ }^{21}$ The hybridization energy required to form $s p^{3}$ orbitals, giving the tetrahedral bonding characteristic of $\mathrm{Si}$, is very high, and structures allowing $p^{3}$ bonding (as in native $\mathrm{Bi}$ bulk) will be more stable. Density of states (DOS) plots for the $\mathrm{Bi}-\mathrm{V}$ structure show that the $\mathrm{Bi} 6 s$ orbital can almost be considered as a part of the core, with only the $6 p$ electrons forming valence bonds. Two of the three $6 p$ orbitals form bonds with second-layer $\mathrm{Si}$ atoms, and the final $6 p$ orbital remains unbonded and half-filled, aligned in the plane of the surface and perpendicular to the $\mathrm{Si}-\mathrm{Si}$ dimer row direction as shown in Fig. 1(c). The DOS of the Bi thus originates mainly from $6 p$ orbitals, and the contributions from $s$ and $d$ orbitals are small in the energy range studied here.

Of the structures which we have modeled covering the possible atomic arrangements, only the $\mathrm{Bi}-\mathrm{V}$ structure matches the bias-dependent STM images and spectroscopy. Of all structures involving vacancies, DFT finds the symmetric $\mathrm{Bi}$ structure to be most stable, with the $\mathrm{Bi}-\mathrm{V}$ structure the next most stable; however, a hybrid DFT calculation finds that this ordering is reversed, with the $\mathrm{Bi}-\mathrm{V}$ structure most stable (see below). A possible formation mechanism for the $\mathrm{Bi}-\mathrm{V}$ structures at the surface is the diffusion of $\mathrm{Bi}$ atoms through the bulk in association with $\mathrm{Si}$ vacancies. ${ }^{22}$ Further DFT calculations, also detailed below, indeed show that the $\mathrm{Bi}$ and $\mathrm{Si}$ vacancy will remain associated at the surface, once paired.

We give the total energies of various structures in Table I, from both DFT GGA and hybrid DFT calculations, as DFT will underestimate the energy of localized states thus also underestimating the stability of features such as the Bi-V. The structures modeled are illustrated in Fig. 4. The energies are all calculated for unit cells with the same number of atoms (hence we have included a surface vacancy in the calculations where $\mathrm{Bi}$ is not associated with a vacancy).

It is important to note various points about the energies. First, once a vacancy is present at the surface, the Bi-V or symmetric $\mathrm{Bi}$ structures are most stable, as shown in Table I. Second, given the nonequilibrium preparation technique, structures which are energetically unfavorable can form because kinetic pathways derived from the highly nonequilibrium ion implantation process and subsequent incomplete

TABLE I. Energies calculated with plane wave and Gaussian basis set spin-polarized DFT of possible structures relative to $\mathrm{Bi}$ atom placed symmetrically in missing dimer vacancy (symmetric $\mathrm{Bi})$. For symmetric $\mathrm{Bi}$ and $\mathrm{Bi}-\mathrm{V}$ the energies relative to symmetric $\mathrm{Bi}$ calculated with hybrid DFT are also shown. Structures are illustrated in Fig. 4.

\begin{tabular}{lcc}
\hline \hline Structure & DFT $(\mathrm{eV})$ & Hybrid DFT (B3LYP) (eV) \\
\hline Symmetric Bi & 0.00 & 0.00 \\
Bi-V & 0.39 & -0.04 \\
Bi adatom + 1DV & 0.67 & \\
Bi-Si HD + 1DV & 0.79 & \\
\hline \hline
\end{tabular}




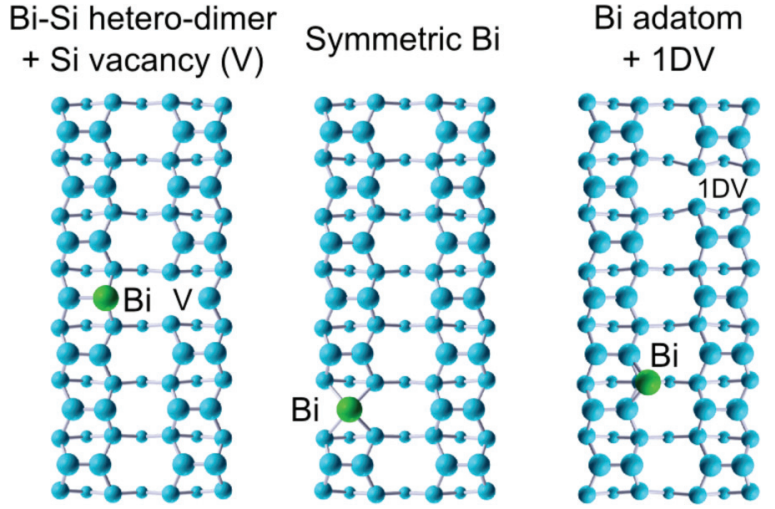

FIG. 4. (Color online) Schematic structures of arrangements of $\mathrm{Bi}$ and vacancies on $\mathrm{Si}(001)$. "1DV" is the well-known single Si dimer vacancy, and "V" is a single Si vacancy.

thermal annealing steps favor their formation. Third, while the symmetric Bi feature has a lower energy, it does not match the feature observed in STM. Fourth, calculations with hybrid functionals indicate that, for the B3LYP functional, the Bi-V structure is more stable than the symmetric $\mathrm{Bi}^{23}$ Finally, significant numbers of energetically unfavorable structures (e.g., defects, steps) are found in clean, well-prepared $\mathrm{Si}(001)$ surfaces in all experiments.

\section{B. Electronic structure}

To understand the electronic structure of the $\mathrm{Bi}-\mathrm{V}$ feature, we performed STS measurements and compared them to non-spin-polarized and spin-polarized DFT DOS calculations. Figure 5(a) shows the differential conductance (dI/dV) spectrum collected with the STM, both at the Bi site and over a Si dimer far from the Bi. The simulated local density of Kohn-Sham states (LDOS) around the Bi dopant and the upper $\mathrm{Si}$ atom of a buckled $\mathrm{Si}-\mathrm{Si}$ dimer far from the Bi site are shown for non-spin-polarized calculations in Figs. 5(c) and 5(d) and for spin-polarized calculations in Figs. 5(e) and 5(f). Note that the non-spin-polarized LDOS are very different to the experiment, while the spin-polarized agree well (discussed in more detail below). Our key result is a double-peaked $\mathrm{Bi}$-induced resonance in the Si dimer gap, visible in both the spin-polarized DFT and STM.

We compare experiment with theory first for clean $\mathrm{Si}$. The $\mathrm{d} / \mathrm{dV}$ spectrum taken at the Si dimer shows characteristic peaks at $\sim-0.8,+0.6 \mathrm{~V}$ [Fig. 5(a)], which are in agreement with previously reported results. ${ }^{4,5}$ Similar features representing $\pi(-0.4 \mathrm{~V})$ and $\pi^{\star}(+0.2 \mathrm{~V})$ states of the Si-Si dimer are seen in the simulated LDOS plot [Figs. 5(d) and 5(f)]. It is well known that DFT significantly underestimates band gaps, though it will give reasonable state densities and charge density distributions once appropriate energy shifts are made. ${ }^{24}$ This will contribute strongly to the energy differences between experiment and theory: $\sim 0.4 \mathrm{~V}$ in both empty and filled states. Another factor is likely to be tip-induced band bending. ${ }^{25}$

The experimental $\mathrm{dI} / \mathrm{dV}$ spectrum of $\mathrm{Bi}$ in Fig. 5(a) shows a sharp peak at $\sim-0.4 \mathrm{~V}$ and a small peak structure at $\sim+0.7 \mathrm{~V}$. The $-0.4 \mathrm{~V}$ peak can be attributed to the sharp peak at $-0.15 \mathrm{~V}$ in the simulated spin-polarized DOS [Fig. 5(e)], associated with the Bi $p$ state. Similarly, the peak at
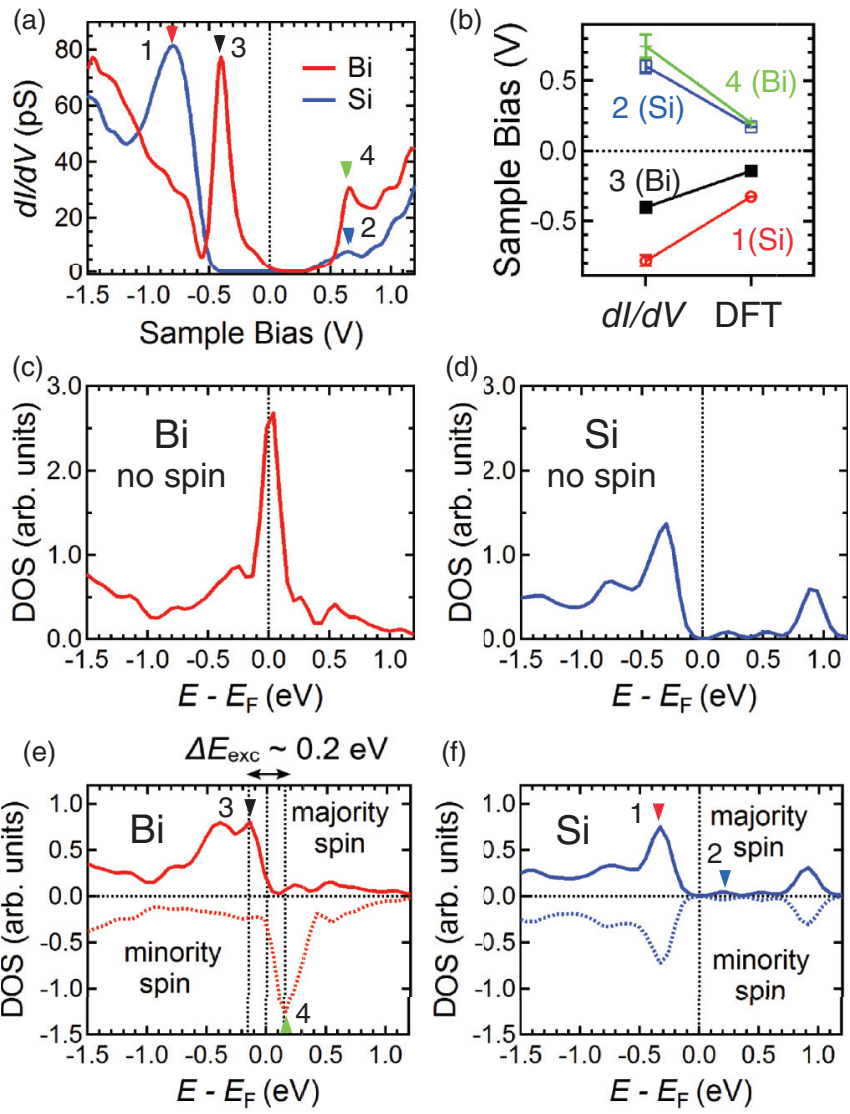

FIG. 5. (Color online) Comparison between STS and both nonspin-polarized and spin-polarized DFT simulated density of states (DOS) of Bi-Si vacancy complex. (a) Differential conductance $\mathrm{dI} / \mathrm{dV}$ at $\mathrm{Bi}$ donor and $\mathrm{Si}$ dimer far from Bi. Characteristic peaks are indicated by arrows and numbers. (b) Comparison of peak bias-voltages between $\mathrm{dI} / \mathrm{dV}$ and spin-polarized calculated DOS. (c) DOS of Bi atom calculated by non-spin-polarized DFT. (d) Simulated DOS of Si far from Bi by non-spin-polarized DFT. (e) Spin-polarized DOS of Bi atom embedded in the supercell shown in Fig. 1(c). The calculated exchange energy is $\sim 0.2 \mathrm{eV}$. Peaks are indicated with the same colors and numbers as for dI/dV. (f) Spin-polarized DOS of $\mathrm{Si}$ far away from Bi.

$+0.7 \mathrm{~V}$ in the $\mathrm{dI} / \mathrm{dV}$ spectrum can be matched to the $+0.15 \mathrm{~V}$ peak in the calculated spin-polarized DOS. The extra peaks seen in the calculated DOS are caused by slight mixing with neighboring $\mathrm{Si}$ atoms and are due to the method of projecting the DOS onto individual atoms. Figure 5(b) summarizes the comparison of peak bias voltages found for $\mathrm{dI} / \mathrm{dV}$ and calculated DOS.

Figure 3(b) shows $\mathrm{dI} / \mathrm{dV}$ maps around the $\mathrm{Bi}-\mathrm{V}$ complex at two characteristic peak energies, $-0.4 \mathrm{~V}$ and $+0.7 \mathrm{~V}$. The filled state map at $-0.4 \mathrm{~V}$ reveals the spatial structure of the $\mathrm{Bi}$ donor level. The DOS around Bi becomes larger reflecting the sharp Bi $p$ state peak, and a substantial hybridization with neighboring $\mathrm{Si}$ atoms can also be observed. In the empty states at $+0.7 \mathrm{~V}$, the $\mathrm{Bi}$ site appears as a localized protrusion. To compare the experimental results with DFT calculations, including the energy shifts due to DFT errors and band bending, we have shifted the simulated DOS map in accord with the shifts of the key spectral features in Fig. 5(a). 
Thus, the simulated DOS map at $-0.15 \mathrm{~V}$ (corresponding to $-0.4 \mathrm{~V}$ in $\mathrm{dI} / \mathrm{dV}$ map) can account for the spatial structure of the $\mathrm{Bi}$ donor level observed in the $\mathrm{dI} / \mathrm{dV}$ map. Also, the localized bright spot in the $\mathrm{dI} / \mathrm{dV}$ map at $+0.7 \mathrm{~V}$ is clearly reproduced at $+0.15 \mathrm{~V}$ in the DFT calculation. The electronic structure of a $\mathrm{Bi}-\mathrm{Si}$ heterodimer is shown in Appendix, and confirms that the $\mathrm{Bi}-\mathrm{V}$ feature is not a heterodimer.

\section{Spin and magnetic structure}

Figures 5(c)-5(f) present non-spin-polarized and polarized densities of states (DOS) for $\mathrm{Bi}$ and $\mathrm{Si}$ atoms. Without polarization, which is required unless the $\mathrm{Bi}$ is ionized, the calculated DOS of $\mathrm{Bi}$ is inconsistent with the measured $\mathrm{dI} / \mathrm{dV}$ spectra, since a single peak at $\mathrm{E}_{F}$ is predicted [Fig. 5(c)]. Taking spin into account, the majority band associated with the unbonded $\mathrm{Bi} p$ state is full and the minority band empty [Fig. 5(e), left], implying full polarization, with a moment of $1 \mu_{B} / \mathrm{Bi}$, and the theory bears much closer resemblance to the data in Fig. 5(a). The associated spin density is dominated by the $\mathrm{Bi}$ atom and one of the up $\mathrm{Si}$ atoms neighboring the Si vacancy on the opposite side of the dimer row, but shows some delocalization along the row. There is no significant difference between polarized and non-spin-polarized results [Figs. 5(d) and 5(f)] for the DOS at the Si sites far from the defect. Thus, the peaks seen in the dI/dV spectra for Bi-V must be due to a spin-polarized Bi state. The splitting between the occupied and unoccupied states at a single spin-polarized defect is approximately $0.2 \mathrm{eV}$, a large value supported by our experiments.

Though we are unable at present to show experimental data in support of this, we now turn to calculations of the energetics of magnetic ordering between pairs of $\mathrm{Bi}-\mathrm{V}$ features. We compare the ferromagnetic and broken-symmetry antiferromagnetic total energies in order to obtain the couplings of the Bi-V pairs. Because of the $p^{3}$ bonding and the size of the atoms, the $\mathrm{Bi}$ atoms will always be found substituting for the "up" position of a buckled dimer, with the vacancy at the "down" site. We have computed only pairs where the $\mathrm{Bi}$ atoms are located on the same side of the dimer row; because of the "up-down" tilt sequence found at low temperatures in the $\operatorname{Si}(001)-c(4 \times 2)$ structure, this implies a spacing of an even number of dimer separations (and hence an odd number of dimers separating the two defect sites).

DFT relies on a finite supercell, with two rows of eight dimers each, to obtain the DOS. It is natural to ask to what extent the splitting is derived from the ferromagnetism implied by the periodic boundary conditions. Accordingly, we have also considered the electronic structure of arrays of pairs of the Bi-V feature in the same supercell with different separations, shown in Fig. 6. For these pairs we can estimate the exchange interaction between neighboring $\mathrm{Bi}$ atoms by calculating the energy difference between ferromagnetic (FM) and antiferromagnetic (AFM) arrangements of the spins localized on the Bi atoms. Since it is known that conventional DFT tends to delocalize electronic states artificially, ${ }^{26}$ and hence overestimate such magnetic
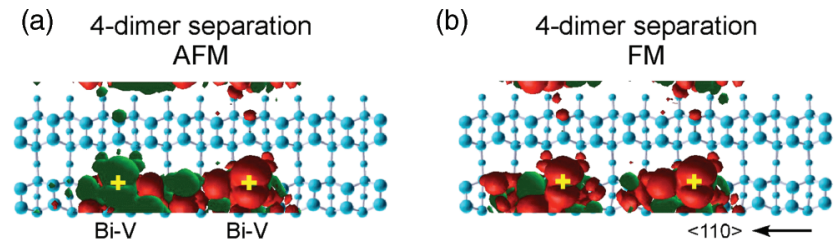

(c) 6-dimer separation

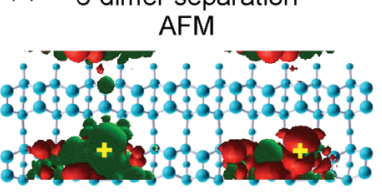

(e) 8-dimer separation AFM

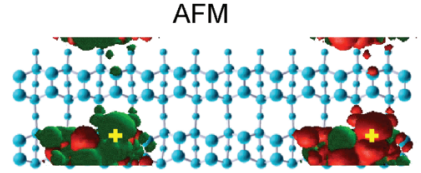

(d) 6-dimer separation FM

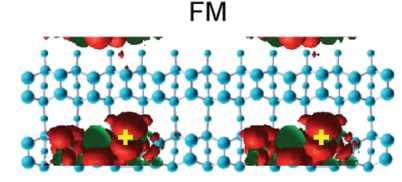

(f)

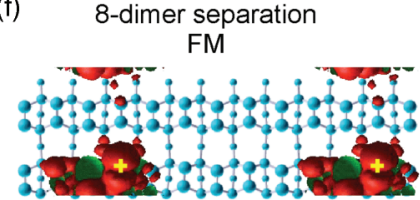

FIG. 6. (Color online) Contours of constant spin density for broken-symmetry AFM configurations (left column) and FM configurations (right column) of $\mathrm{Bi}-\mathrm{V}$ pairs on $\mathrm{Si}(001)$, computed using hybrid DFT. The dimer rows run from left to right; red and blue isosurfaces show positive and negative spin densities, respectively. (a),(b): spacing of four dimers. (c),(d): spacing of six dimers. (e),(f): spacing of eight dimers.

interactions, we have also made this comparison using hybrid DFT.

The calculations considered interactions when $\mathrm{Bi}$ atoms are in the same row, using two rows of sixteen dimers each, with the size constrained by the limits of DFT; all results are shown in Fig. 6. We consider $\mathrm{Bi}$ atoms on the same side of the row, with spacings of 4,6 , or 8 dimers, or $1.54,2.30$, and $3.07 \mathrm{~nm}$. The FM/AFM energy differences derived from hybrid DFT are: $-0.03 \mathrm{eV}$ for $1.54 \mathrm{~nm}$ (4 dimers); they also favor ferromagnetism but have magnitudes below $0.001 \mathrm{eV}$ for 2.30 and $3.07 \mathrm{~nm}$ (6 and 8 dimers). This difference is significant for the 4-dimer spacing, but falls below the accuracy of our calculations for 6 and 8 dimers. The calculations indicate that the interactions are ferromagnetic, in contrast to the antiferromagnetic interaction of around $+0.06 \mathrm{eV}$ estimated for $\mathrm{Bi}$ at the same separation in bulk $\mathrm{Si},{ }^{27}$ with considerable overlap of the associated Rydberg atoms. The difference is a result of the bonding of the $\mathrm{Bi}$ and hence its electronic structure.

There are two significant difficulties in analyzing such magnetic couplings between defect pairs using DFT: first, conventional DFT tends to delocalize artificially localized spin densities, and hence overestimate magnetic couplings. ${ }^{26}$ Second, even in pairs on the insulating side of a Mott transition, the Kohn-Sham orbitals form delocalized molecular states over both members of the pair and the bonding state is doubly occupied in the case of antialigned spins (antiferromagnetic pair), whereas for aligned spins both bonding and antibonding states are singly occupied. This artificially raises the energy of AFM relative to FM structures. We solve the first problem by using the B3LYP hybrid functional, ${ }^{28}$ which adds an empirical mixture of exact exchange into gradient-corrected DFT; this is found to give a better balance between localization 
and delocalization and to give magnetic couplings in better agreement with experiment in a range of structures. ${ }^{26,28} \mathrm{We}$ deal with the second problem by using a broken-symmetry approach, ${ }^{29}$ in which the Kohn-Sham states are no longer required to transform according to irreducible representations of the structural symmetry group but are rotated so as to localize them on the magnetic centres and enable a fair comparison of the FM and AFM configurations.

The resulting spin densities are shown in Fig. 6. The strong two-lobed feature corresponding to the unbonded $\mathrm{Bi} p$ state is clearly visible in all cases, as is the delocalization of the spin density onto the dangling bond for a neighboring Si up-atom. In most cases this is on the opposite side of the dimer row, but for the smallest separation considered (4 dimer spacings) the strain in the surface has caused the neighboring dimer of the right-hand defect shown to reverse its tilt, and accordingly the spin density spills onto the neighboring $\mathrm{Si}$ on the same side of the dimer row instead. The origin of the magnetic coupling in the delocalization of the spin density along the dimer row is clearly visible in the 4-dimer spacing case, and can also be seen in lower-density isosurfaces (not shown) for the larger separations.

The localization of the $\mathrm{Bi}$ state on the surface is significantly greater than in bulk, but it can couple to the delocalized $\pi^{\star}$ state on the surface along dimer rows; in the limit of no dimer tilt, where the surface bands are half-filled and therefore metallic, the resulting RKKY interaction will give alternating ferromagnetic and antiferromagnetic interactions along a row.

\section{CONCLUSION}

We have discovered an unusual structure formed by the heavy dopant $\mathrm{Bi}$ at $\mathrm{Si}(001)$ surfaces following ion-implantation and surface cleaning. Figure 7 , where the

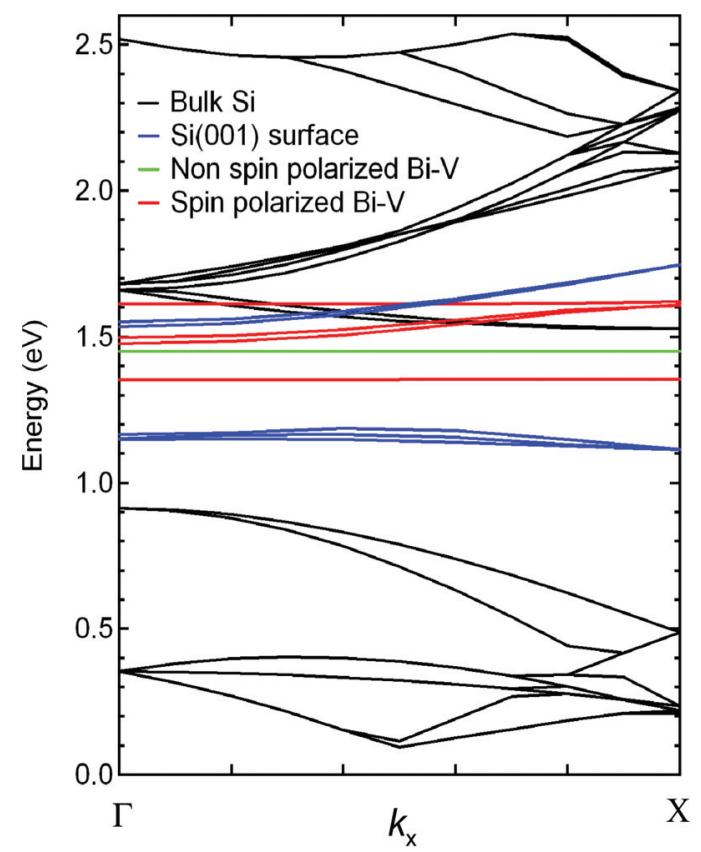

FIG. 7. (Color online) Band structure of bulk silicon, $\operatorname{Si}(001)$, and $\mathrm{Bi}-\mathrm{V}$ feature along $\Gamma-\mathrm{X}(100)$ direction in the conventional cubic cell. band structures for bulk $\mathrm{Si}, \mathrm{Si}(001)$, and the $\mathrm{Bi}-\mathrm{V}$ feature, with and without spin polarization, are plotted, shows the essential physics. The reduction of the band gap with creation of the surface is clear, along with the introduction of defect levels in the surface state gap. The on-site exchange, due to the Coulomb interaction, splits majority from minority spins and opens up a large gap for the $\mathrm{Bi}$ levels. $\mathrm{Bi}$ thus behaves quite unlike a bulk dopant, or even a conventional light dopant (e.g., P) at the surface. We understand the formation and nature of this structure, and its absence for lighter dopants, as the natural consequence of the much less significant bonding role of the outer $s$ orbital for a heavy atom in column $\mathrm{V}$ of the periodic table. Beyond the new atomic arrangement associated with the incorporation of $\mathrm{Bi}$ in the surface, the electronic structure is unprecedented for a dopant at the surface of Si: The Bi atom has a half-filled, localized orbital, which carries an unpaired spin. Given the interest in ferromagnets based on semiconductors, it is especially encouraging that the DFT results show that superlattices of $\mathrm{Bi}-\mathrm{V}$ defects favor ferromagnetic order, and indicate exchange couplings as large as $0.03 \mathrm{eV}$ between surface Bi atoms. For the future, the calculations suggest that arrays of closely-spaced $\mathrm{Bi}-\mathrm{V}$ features would be fully polarized at accessible temperatures. This would represent progress over a variety of heterogeneous thin film systems ${ }^{30,31}$ including $\mathrm{Si}$ as well as dilute magnetic semiconductors such as $\mathrm{Mn}$ in $\mathrm{GaAs}^{32,33}$ and Ge. ${ }^{34}$ The half-filled orbitals which we have discovered provide an entrée to $\mathrm{Si}(100)$ surface-based quantum many-body physics, thus complementing previous work ${ }^{35}$ concerning adatom layers on $\mathrm{Si}(111)$, and could also find use in surface spin-based quantum information technology. ${ }^{36,37}$

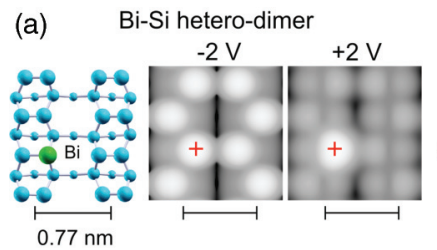

(b)
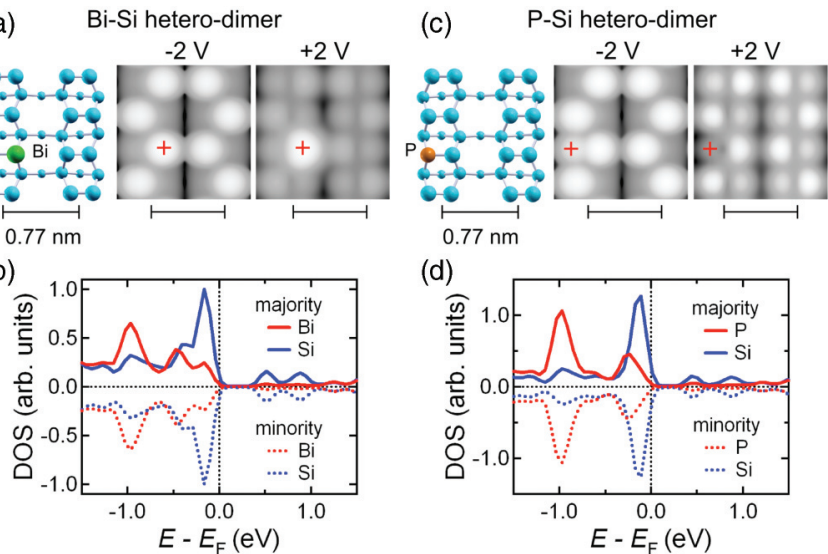

(d)

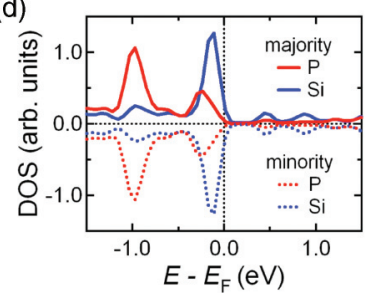

FIG. 8. (Color online) Simulated integrated DOS images and DOS of $\mathrm{Bi}-\mathrm{Si}$ and $\mathrm{P}-\mathrm{Si}$ hetero-dimers in $\mathrm{Si}(001)$, with the $\mathrm{Bi}$ and $\mathrm{P}$ atoms replacing the upper atom of a $\mathrm{Si}$ dimer in $\mathrm{c}(4 \times 2)$ reconstruction. (a) Left: Schematic structure of $\mathrm{Bi}-\mathrm{Si}$ hetero-dimer. The Bi atom has a neutral configuration. Right: Simulated integrated DOS images at $\mathrm{Vs}=-2 \mathrm{~V},+2 \mathrm{~V}$. Bi sites are shown by markers. (b) Spin-polarized DOS of $\mathrm{Bi}$ and $\mathrm{Si}$ atoms in the Bi-Si hetero-dimer structure shown in (a). (c) Left: Schematic structure of P-Si heterodimer. Right: Simulated integrated DOS images at Vs $=-2 \mathrm{~V},+2 \mathrm{~V}$, without the additional electron discussed in Ref. 16. The P sites are marked by crosses ' + '. Both images are for isosurface values of 0.1 electron $/ \mathrm{nm}^{3}$. (d) Spin-polarized DOS of $\mathrm{P}$ and $\mathrm{Si}$ atoms in the P-Si hetero-dimer structure. All integrated DOS images are for isosurface values of 0.1 electron $/ \AA^{3}$. 


\section{ACKNOWLEDGMENTS}

This work was supported by the European Union, the Basic Technologies Programme of the RCUK, a Wolfson-Royal Society Research Merit Award, the EPSRC-funded programme COMPASSS, a Royal Society URF, and the Brazilian agencies CNPq and FAPESB. We are also very grateful to N. Curson, S. Schofield, C. Hirjibehedin, A. Fischer, J. P. de Souza, and H. Boudinov for numerous helpful discussions.

\section{APPENDIX: COMPARISON TO HETERODIMERS}

As a further test of the correct assignment of the Bi-V structure to the observed features, we have simulated STM and STS data for the Bi-Si heterodimer, and compare it to the P-Si heterodimer in Fig. 8. It is clear that this structure is not what is observed in STM; it is also interesting to note that the STS appearance for both $\mathrm{P}$ and $\mathrm{Bi}$ is extremely similar.
*Present address: Center for Emergent Matter Science, RIKEN, Saitama 351-0198, Japan.

${ }^{1}$ P. Studer, V. Brázdová, S. R. Schofield, D. R. Bowler, C. F. Hirjibehedin, and N. J. Curson, ACS Nano 6, 10456 (2012). ${ }^{2}$ G. W. Morley, M. Warner, A. M. Stoneham, P. T. Greenland, J. van Tol, C. W. M. Kay, and G. Aeppli, Nat. Mater. 9, 725 (2010).

${ }^{3}$ E. Abramof, A. Ferreira da Silva, B. E. Sernelius, J. P. de Souza, and H. Boudinov, Phys. Rev. B 55, 9584 (1997).

${ }^{4}$ S. Yoshida, T. Kimura, O. Takeuchi, K. Hata, H. Oigawa, T. Nagamura, H. Sakama, and H. Shigekawa, Phys. Rev. B 70, 235411 (2004).

${ }^{5}$ K. Sagisaka and D. Fujita, Phys. Rev. B 71, 245319 (2005).

${ }^{6}$ K. Hata, S. Ozawa, Y. Sainoo, K. Miyake, and H. Shigekawa, Surf. Sci. 447, 156 (2000).

${ }^{7}$ R. J. Hamers and U. K. Kohler, J. Vac. Sci. Technol. A 7, 2854 (1989).

${ }^{8}$ G. W. Brown, H. Grube, M. E. Hawley, S. R. Schofield, N. J. Curson, M. Y. Simmons, and R. G. Clark, J. Appl. Phys. 92, 820 (2002).

${ }^{9}$ Z. Zhang, M. A. Kulakov, B. Bullemer, I. Eisele, and A. V. Zotov, J. Vac. Sci. Technol. B 14, 2684 (1996).

${ }^{10}$ J. P. Perdew, K. Burke, and M. Ernzerhof, Phys. Rev. Lett. 77, 3865 (1996).

${ }^{11}$ G. Kresse and J. Furthmuller, Phys. Rev. B 54, 11169 (1996).

${ }^{12}$ D. Vanderbilt, Phys. Rev. B 41, 7892 (1990).

${ }^{13}$ R. Dovesi, V. Saunders, C. Roetti, R. Orlando, C. M. Zicovich-Wilson, F. Pascale, B. Civalleri, K. Doll, N. Harrison, I. Bush et al., CRYSTALO6 User's Manual, University of Torino, Torino (2006).

${ }^{14}$ W. Watd and P. Hay, J. Chem. Phys. 82, 284 (1985).

${ }^{15}$ R. M. Tromp, R. J. Hamers, and J. E. Demuth, Phys. Rev. Lett. 55, 1303 (1985).

${ }^{16}$ M. W. Radny, P. V. Smith, T. C. G. Reusch, O. Warschkow, N. A. Marks, H. F. Wilson, N. J. Curson, S. R. Schofield, D. R. McKenzie, and M. Y. Simmons, Phys. Rev. B 74, 113311 (2006).

${ }^{17}$ T. C. G. Reusch, M. W. Radny, P. V. Smith, O. Warschkow, N. A. Marks, N. J. Curson, D. R. McKenzie, and M. Y. Simmons, J. Phys. Chem. C 111, 6428 (2007).
${ }^{18}$ T. Reusch, N. Curson, S. Schofield, T. Hallam, and M. Simmons, Surf. Sci. 600, 318 (2006).

${ }^{19}$ N. Itoh and A. M. Stoneham, Materials Modification by Electronic Excitation (Cambridge University Press, Cambridge, 2000).

${ }^{20}$ J. H. G. Owen, K. Miki, H. Koh, H. W. Yeom, and D. R. Bowler, Phys. Rev. Lett. 88, 226104 (2002).

${ }^{21}$ D. R. Bowler, Phys. Rev. B 62, 7237 (2000).

${ }^{22}$ P. M. Fahey, P. B. Griffin, and J. D. Plummer, Rev. Mod. Phys. 61, 289 (1989).

${ }^{23}$ The precise ordering of the structures is dependent on the effective core potential and the basis set used as well as on the exchangecorrelation potential.

${ }^{24}$ R. M. Martin, Electronic Structure: Basic Theory and Practical Methods (Cambridge University Press, Cambridge, 2004).

${ }^{25}$ R. M. Feenstra, G. Meyer, F. Moresco, and K. H. Rieder, Phys. Rev. B 66, 165204 (2002).

${ }^{26}$ A. Zunger, S. Lany, and H. Raebiger, Nature Phys. 3, 53 (2010).

${ }^{27}$ W. Wu and A. J. Fisher, Phys. Rev. B 77, 045201 (2008).

${ }^{28}$ A. D. Becke, J. Chem. Phys. 98, 5648 (1993).

${ }^{29}$ L. Noodleman, J. Chem. Phys. 74, 5737 (1981).

${ }^{30}$ A. Schmehl, V. Vaithyanathan, A. Herrnberger, S. Thiel, C. Richter, M. Liberati, T. Heeg, M. Rockerath, L. F. Kourkoutis, S. Muhlbauer et al., Nat. Mater. 6, 882 (2007).

${ }^{31}$ B. T. Jonker, G. Kioseoglou, A. T. Hanbicki, C. H. Li, and P. E. Thompson, Nature Phys. 3, 542 (2007).

${ }^{32}$ H. Ohno, Science 281, 951 (1998).

${ }^{33}$ D. Kitchen, A. Richardella, J.-M. Tang, M. E. Flatté, and A. Yazdani, Nature 442, 436 (2006).

${ }^{34}$ Y. D. Park, A. T. Hanbicki, S. C. Erwin, C. S. Hellberg, J. M. Sullivan, J. M. Sullivan, J. M. Sullivan, J. E. Mattson, T. F. Ambrose, A. Wilson, G. Spanos, and B. T. Jonker, Science 295, 651 (2002).

${ }^{35}$ G. Santoro, S. Scandolo, and E. Tossati, Phys. Rev. B 59, 1891 (1999).

${ }^{36}$ A. M. Stoneham, A. J. Fisher, and P. T. Greenland, J. Phys.: Condens. Matter 15, L447 (2003).

${ }^{37}$ B. E. Kane, Nature 393, 133 (1998). 